\title{
Ethnicity and risk of cardiovascular disease (CVD): 4.8 year follow-up of patients with type 2 diabetes living in Scotland
}

\author{
Muhammad Omar Malik • L. Govan • John R. Petrie • Nazim Ghouri • \\ Graham Leese • Colin Fischbacher • Helen Colhoun • Sam Philip • \\ Sarah Wild • Rory McCrimmon • Naveed Sattar • Robert S. Lindsay • \\ on behalf of Scottish Diabetes Research Network (SDRN) Epidemiology group
}

Received: 24 September 2014 / Accepted: 22 December 2014 /Published online: 12 February 2015

(C) Springer-Verlag Berlin Heidelberg 2015

\begin{abstract}
Aims/hypothesis Potential differences in cardiovascular risk by ethnicity remain uncertain. We evaluated the association of ethnicity with cardiovascular disease (CVD) incidence in a large cohort of people with type 2 diabetes living in Scotland.

Methods Data from Scottish Care Information-Diabetes (SCIDiabetes) were linked to Scottish Morbidity Records (SMR01) and National Records of Scotland data for mortality for dates between 2005 and 2011. Of 156,991 people with type 2 diabetes with coded ethnicity, 121,535 (77.4\%) had no CVD at baseline (White: 114,461; Multiple Ethnic: 2, 554; Indian: 797; Other Asian: 319; Pakistani: 2,250; Chinese: 387; African-Caribbean: 301 and Other: 466) and were followed up (mean \pm SD: $4.8 \pm 2.3$ years) for the development of fatal and non-fatal CVD.

Results During follow-up, 16,265 (13.4\%) patients developed CVD (ischaemic heart or cerebrovascular

Electronic supplementary material The online version of this article (doi:10.1007/s00125-015-3492-0) contains peer-reviewed but unedited supplementary material, which is available to authorised users.
\end{abstract}

M. O. Malik · J. R. Petrie • N. Ghouri • N. Sattar · R. S. Lindsay $(\triangle)$ Institute of Cardiovascular and Medical Sciences, BHF Glasgow,

Cardiovascular Research Centre, University of Glasgow,

126 University Place, G12 8TA Glasgow, UK

e-mail: robert.lindsay@glasgow.ac.uk

L. Govan

Health Economics and Health Technology Assessment, Institute of

Health and Wellbeing, University of Glasgow, Glasgow, UK

G. Leese

Ninewells Hospital and Medical School Dundee, Dundee, UK

C. Fischbacher

Information Services Division (ISD), NHS National Services

Scotland, Edinburgh, UK diseases). At baseline, Pakistanis were younger and had developed diabetes earlier, had higher $\mathrm{HbA}_{1 \mathrm{c}}$ and longer duration of diabetes, but had lower BP, BMI, creatinine, proportion of smokers and proportion on antihypertensive therapy than whites. The age and sex adjusted HRs for CVD were HR 1.31 (CI 1.17, 1.47), $p<0.001$ in Pakistanis and HR 0.66 (CI 0.47, 0.92), $p=$ 0.014 in Chinese compared with whites. Adjusting additionally for an area measure of deprivation, duration of diabetes, conventional CVD and other risk factors, the HR for Pakistanis (HR 1.45 [CI 1.14, 1.85], $p=0.002$ ) was significantly higher, and that for Chinese $(\mathrm{HR}=0.58$ [CI $0.24,1.40], p=0.228)$ lower, compared with whites.

Conclusions/interpretation Compared with whites with type 2 diabetes, those of Pakistani ethnicity in Scotland were at increased risk of CVD, whereas Chinese were at lower risk, with these differences unexplained by known risk factors.

H. Colhoun

School of Medicine, The Mackenzie Building, University of Dundee, Dundee, UK

S. Philip

Diabetes and Endocrinology, Aberdeen Royal Infirmary,

Aberdeen, UK

S. Wild

Centre for Population Health Sciences, University of Edinburgh, Edinburgh, UK

R. McCrimmon

Medical Research Institute, University of Dundee, Dundee, UK 
Keywords Cardiovascular disease (CVD) - Cox-regression . Ethnicity $\cdot \mathrm{HbA}_{1 \mathrm{c}} \cdot$ Longitudinal $\cdot$ Pakistani $\cdot$ Renal failure Type 2 diabetes · White

$\begin{array}{ll}\text { Abbreviations } & \\ \text { BNF } & \text { British National Formulary } \\ \text { CVD } & \text { Cardiovascular disease } \\ \text { HDL-c } & \text { HDL-cholesterol } \\ \text { LDL-c } & \text { LDL-cholesterol } \\ \text { MI } & \text { Myocardial infarction } \\ \text { NHS } & \text { National Health Service } \\ \text { SCI-Diabetes } & \text { Scottish Care Information-Diabetes } \\ \text { SDRN } & \text { Scottish Diabetes Research Network } \\ \text { SES } & \text { Socioeconomic status } \\ \text { SIMD } & \text { Scottish Index of Multiple Deprivation } \\ \text { SMR01 } & \text { Scottish Morbidity Records } \\ \text { TG } & \text { Triacylglycerol }\end{array}$

\section{Introduction}

Type 2 diabetes is associated with an increased risk of premature mortality, and cardiovascular disease (CVD) is the most common cause of death [1]. More than $60 \%$ of people with type 2 diabetes die of CVD, predominantly myocardial infarction (MI) or stroke [2]. In developed countries, rates of cardiovascular death are decreasing, but for people of South Asian origin living in those countries, the rates are stable [3], or even increasing, in comparison with white individuals living in those countries $[4,5]$.

South Asians are at an increased risk of CVD compared with their white counterparts [6,7]; cardiovascular risk factors are increased with higher waist:hip ratio $[8,9]$ and an increased risk of diabetes [7, 10], which has an earlier onset $[11,12]$ and a greater likelihood of suboptimal glycaemic control $[13,14]$. In the UK, excess CVD in South Asians is in part explained by higher rates of diabetes [15]. However, even within those with diabetes, the rate of CHD deaths is double that in whites and does not appear to be explained by conventional risk factors - albeit in relatively small numbers (311 South Asians, 51 deaths) [8]. South Asians are considered a single group in most of the studies $[7,8,15]$, despite the fact that ethnic differences in risk of CVD within South Asian countries have been reported [5, 16-19].

We have investigated whether there are ethnic differences in rates of CVD in a large prospective cohort of people with clinically diagnosed diabetes, and further, whether any differences are independent of (or explained by) conventional CVD risk factors including the onset, duration and severity of diabetes.

\section{Methods}

Population-based data are available for people with diagnosed diabetes in Scotland in the Scottish Care Information-Diabetes (SCI-Diabetes) dataset (previously known as SCI-DC), an electronic patient record of National Health Service (NHS) Scotland patients with diabetes. An extract of SCI-Diabetes data until 31 December 2011 was linked to Scottish Morbidity Records (SMR01) and National Records of Scotland for mortality provided by the National Records of Scotland. The Information Services Division (ISD) Scotland, Data Quality Assurance (DQA) team assesses the accuracy rate and quality of SMR01. For ischaemic heart disease, the accuracy, sensitivity and completeness of SMR01 in the 2012 report were $97.3 \%, 94.8 \%$ and $97.4 \%$, respectively [20]. For cerebrovascular disease, the accuracy, sensitivity and completeness were $94.5 \%, 98.9 \%$ and $100 \%$, respectively [20]. The detailed methodology of quality assessment can be found at www. isdscotland.org/Products-and-Services/Data-Quality/ Methodology/. Data were also linked to the Scottish Index of Multiple Deprivation (SIMD)-2012 data set, which is the Scottish Government's official tool for identifying concentrated areas of multiple deprivations in Scotland. Quintiles of the index are defined at a national level, and Q1 and Q5 were used to identify the most and least deprived quintiles, respectively. (See www.scotland.gov.uk/Topics/ Statistics/SIMD/ for more information.) Ethics approval for the linkage and analysis was obtained from the Caldicott guardians of all 14 Health Boards in Scotland (PAC Approval-33/11 and MREC-Reference: 11/AL/0225).

For the dataset, type of diabetes was confirmed by an algorithm using age at diagnosis and use and timing of treatment with oral hypoglycaemic agents and insulin [21]. Ethnicity information was obtained from SCI-Diabetes based on the fact that people with diabetes are asked to identify their ethnic group from a standard list used in the 2001 Census in Scotland [13]. As numbers were small $(n=91)$, those of Bangladeshi origin were merged with the Other Asian group.

Included in the study were all people with type 2 diabetes having: (1) follow-up data (either hospital admission or any data for outpatient clinical visits) between January 2005 and December 2011; and (2) available ethnicity data. Patients with no follow-up data for clinical variables after $2005(n=4557$, $1.6 \%)$ were excluded on the assumption that they may have left the area (Fig. 1). People with other types of diabetes, missing type of diabetes or who developed type 2 diabetes before the age of $17(n=109)$ were excluded from analysis. Children aged $<17$ were excluded as the SCI-Diabetes system is used mainly for adults and has less complete coverage in the paediatric population. All patients with missing ethnicity data ( $n=67,994,24 \%)$ were also excluded. Patients with inconsistent data $(n=12)$ were also excluded - i.e. dates of examination after death. 


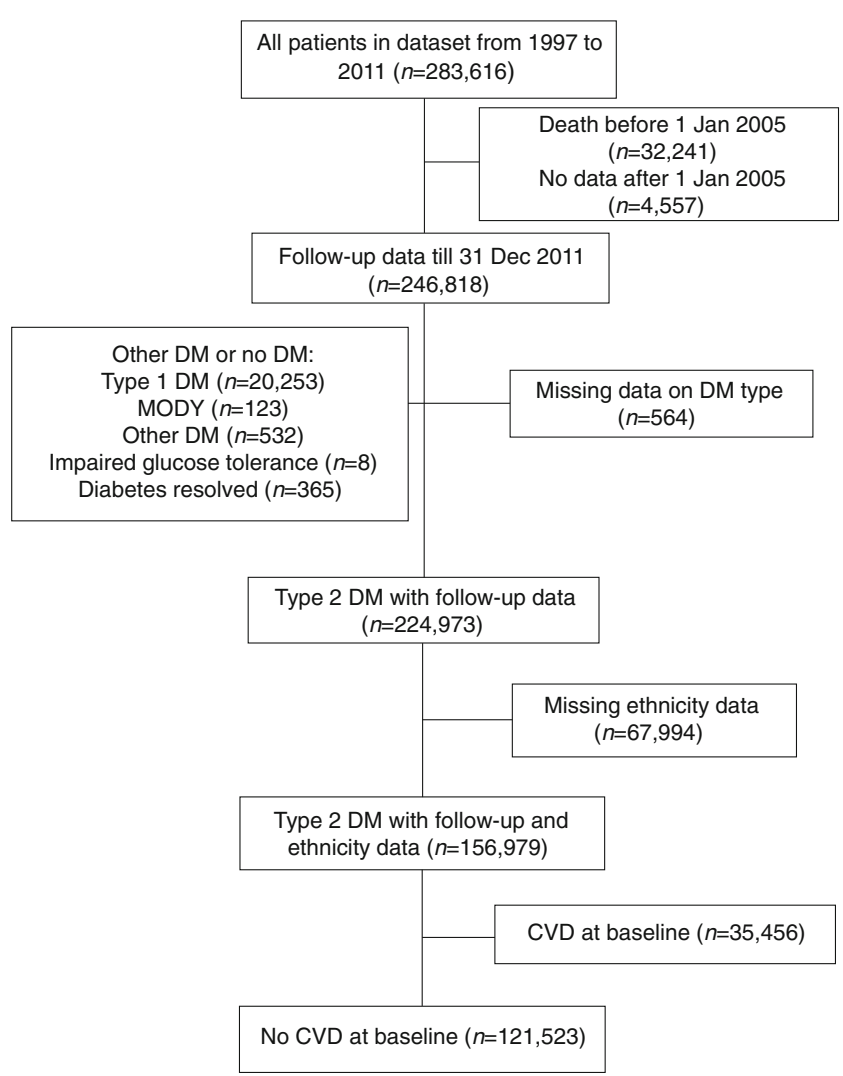

Fig. 1 CVD in people with type 2 diabetes in Scotland: numbers of patients excluded. Dec., December; DM, diabetes mellitus

For the analysis, entry date was taken as 1 January 2005 or the date of diabetes diagnosis if later, and exit date as 31 December 2011 or date of first CVD event or death if earlier. Incident CVD events (cardiovascular and cerebrovascular) were obtained from SMR-01 records using ICD-10: I20-I25, I60-69 (excluding I62 and I68; www.who.int/classifications/ icd/en/). For baseline anthropometric and biochemical measures (BMI, BP, total cholesterol, HDL-cholesterol (HDL-c), LDL-cholesterol (LDL-c), triacylglycerol [TG], $\mathrm{HbA}_{1 \mathrm{c}}$, creatinine), the nearest values preceding the entry date were obtained. Implausible values for the variables were excluded before conducting the analyses.

Prevalent CVD at baseline was defined as hospital admission for CVD (using codes above and equivalent ICD-9 codes [www.icd9data.com/2007/Volume1] for earlier data) in retrospective data to 1992 with similar 'lookback' time for the different ethnic groups (e.g. White: mean \pm SD $4.7 \pm 2.8$ years, Multiple Ethnic: $4.9 \pm 3.0$, Indian: $4.6 \pm 2.5$, Other Asian: $4.7 \pm 2$. 3, Pakistani: $4.2 \pm 2.6$ years, African-Caribbean: $4.2 \pm 2.8$ years and Other Ethnic: $4.7 \pm 2.7$ ). After exclusions, a total of 121,523 people who did not have any history of CVD at baseline were followed for development of CVD (Fig. 1).

Statistical analysis All continuous variables (age at baseline, age at diabetes diagnosis, $\mathrm{BP}, \mathrm{BMI}, \mathrm{HbA}_{1 \mathrm{c}}$, total cholesterol,
HDL-c, LDL-c and creatinine) were normally distributed except for TG and duration of diabetes, which were log transformed for analysis. In univariate analysis, continuous variables were compared across different ethnic groups by ANOVA with Bonferroni adjustment. Categorical variables (incident CVD event, sex, baseline renal impairment, smoking, diabetes treatment, hypertension treatment, high cholesterol treatment and socioeconomic status [SES]) were compared across different ethnic groups by $\chi^{2}$ tests. Logistic regression was used for individual group comparison with White as the reference category. Where previously recorded as a percentage, $\mathrm{HbA}_{1 \mathrm{c}}$ was expressed as International Federation of Clinical Chemistry (IFCC) units. A Cox proportionalhazards regression model was used to examine the relationship of ethnicity and CVD incidence expressed as HRs with corresponding 95\% CIs with and without adjustment for other covariates (age, sex, $\mathrm{HbA}_{1 \mathrm{c}}$, duration of diabetes, SES, severe renal impairment [defined as serum creatinine $\geq 200 \mu \mathrm{mol} / 1$ ], cholesterol lowering treatment [defined as drug use with British National Formulary—BNF code 2.12], antihypertensive treatment [defined as drug use with BNF code 2.5], smoking status, BMI, BP [both systolic and diastolic], total cholesterol and HDL-c). To explore the effects of residual BP and cholesterol on CVD risk, both BP and cholesterol levels were added in the same model along with hypertension and cholesterol lowering treatment. Cox regression was done, both by including and excluding BP and cholesterol in the model. Proportional hazard assumptions were confirmed by minus log-log plot, Kaplan-Meier and predicted survival plot and also by using Schoenfeld residuals. Due to multicollinearity with age $(0.90, p<001)$, age of diabetes diagnosis was omitted from Cox regression. To explore potential effects of ethnicity on the relationship of known cardiovascular risk factors to CVD events further, separate models incorporating interaction terms (sex, $\mathrm{HbA}_{1 \mathrm{c}}$, hypertension treatment, cholesterol treatment, total cholesterol, renal impairment, smoking, age, age at diagnosis and diabetes treatment) were examined. All data analyses were performed using STATA 12 (http://www.stata.com/).

\section{Results}

The differences in anthropometric and metabolic variables, and risk factors, between different ethnic groups and their white counterparts are shown in Table 1. Pakistanis, AfricanCaribbeans and the group designated Other Asians were younger at baseline and their diabetes was diagnosed at a younger age than in whites. Pakistanis, Other Asians and Chinese individuals had lower BP, BMI and total cholesterol than whites. Pakistanis and African-Caribbeans had the highest $\mathrm{HbA}_{1 \mathrm{c}}$ values. Whites and those designated to be of multiple ethnicity had the highest percentage of ever smokers and of 
being on antihypertensive treatment. Pakistanis, Indian and Chinese had the highest mean (geometric) duration of diabetes at baseline and at the end of follow-up (Tables 1 and 2). Whites were the largest group (94.2\%) at baseline (Table 1). Of those excluded from this analysis due to existing CVD, the ethnicity distribution was similar to those included i.e. White (95.2\%), Multiple Ethnic (2\%), Indian (0.5\%), Other Asian (0.14\%), Pakistani $(1.54 \%)$, Chinese $(0.17 \%)$, AfricanCaribbean $(0.10 \%)$ and Other Ethnic (0.28\%).

In total, 16,265 (13.4\%) patients had a CVD event in the follow-up period (Table 2) with ischaemic heart disease the dominant category (electronic supplementary material [ESM] Table 1). After adjustment for age and sex, risk of CVD was increased in Pakistanis (HR 1.31 [CI 1.17, 1.47], $p<0.001$ ) and decreased in Chinese (HR 0.66 [CI 0.47, 0.92], $p=0.014$ ) as compared with whites. Adjustment for other cardiovascular risk factors made little difference to the estimate of HR for Pakistanis: after inclusion of all relevant risk factors Pakistanis remained at higher CVD risk (HR 1.45 [CI 1.14, 1.85], $p=$ 0.002 , Table 3 ), but the results for those of Chinese origin and Other Ethnic groups were no longer significant (Table 3). The risk of CVD was also significant in Pakistani males (HR 1.33 [CI 1.01, 1.75], $p=0.042$ ) and females (HR 1.67 [CI 1.25, 2.24], $p=0.001$ ). The main finding (HR significant for Pakistani population only) remained unchanged whether or not BP was included in the model. Findings were similar for total cholesterol and cholesterol lowering treatment. In addition, removing both BP and total cholesterol did not change the main finding (results not shown). None of the cardiovascular risk factors had significant interactions with ethnicity (sex, $p=$ 0.668; hypertension treatment, $p=0.226$; high cholesterol treatment, $p=0.473$; total cholesterol, $p=0.902 ; \mathrm{HbA}_{1 \mathrm{c}}, p=$ 0.394; renal impairment, $p=0.347$; smoking, $p=0.355$; age, $p=0.441$; age of diabetes onset, $p=0.138$; and diabetes treatment, $p=0.793$ ).

Notably, Pakistanis and Other Asians were almost 9 years younger when they had a CVD event (Table 2). In keeping with this, for the same age and age of diagnosis, Pakistanis had a higher incidence of CVD events as compared with those of white and Chinese origins (Fig. 2).

In total 17,637 people died during the follow-up and deaths due to CVD were 3,722 (21\% of total deaths). The number of deaths due to CVD and other causes in different ethnic groups are shown in ESM Table 1.

\section{Discussion}

In this analysis, we clearly demonstrate increased CVD risk in Pakistanis compared with white individuals with type 2 diabetes living in Scotland. Similar findings have previously been reported in the general population [22-24]; here we have shown this for the first time in a population with diabetes and demonstrated that the excess risk persists even after full statistical adjustment for BMI, metabolic, lifestyle, SES and disease factors. Independent of the effect of age and age of diabetes diagnosis, rates of CVD differed among the various ethnic groups. Once they had developed diabetes, those of Pakistani origin had an increased rate of CVD at all ages compared with white and Chinese individuals with diabetes. Within the same age category, Pakistanis had an increased risk of CVD relative to the white population (HR 1.45 [CI 1.14, $1.85], p=0.002)$ and this was apparent for all age groups over the age of 41 (Fig. 2). Furthermore, Pakistanis with diabetes appear to have the same incidence of CVD approximately 10 years earlier than people in the white or Chinese groups (Fig. 2a).

CVD is one of the leading causes of death in people with type 2 diabetes and more than $60 \%$ of people with type 2 diabetes die of CVD (MI or stroke) [2]. Differences in diabetes incidence rates $[8,12,25]$ and CVD rates $[8,24,26]$ have been evaluated in many studies. Ethnic differences in age of diabetes diagnosis [11, 12, 27], diabetes prevalence [8, 19], $\mathrm{HbA}_{1 \mathrm{c}}$ levels [28, 29], body composition [8, 9, 30], smoking $[28,31]$, physical activity $[12,32,33]$ and $\operatorname{diet}[6,34]$ have all been reported previously. Many studies have also reported effects of SES on diabetes [35, 36] and CVD incidence [37]. The present study adds to this literature in showing an increased risk of CVD in Pakistanis after full adjustment for duration of diabetes and SES. The increased risk of CVD by ethnicity was not dependent on known risk factors; neither was it accounted for by an increased effect of risk factors in the Pakistani group.

South Asians are considered a single group in most studies, and here we report a significant difference only in Pakistanis. Importantly, people of Pakistani origin were different in a number of key characteristics. Previous work from our group has shown that Pakistanis living in Scotland have higher average levels of glycaemia and higher levels of social deprivation than Indians [13]. When Pakistanis were compared with Indians in our cohort, they were younger at baseline (53.8 \pm 12 vs $56.2 \pm 13$ years, respectively; $p<0.001$ ), younger at diabetes diagnosis ( $48.8 \pm 11.6$ vs $51.2 \pm 12.2$ years, respectively; $p<0.001$ ), had higher BMI (30.2 vs 28.8 , respectively; $p<0.001)$, lower BP $(134 / 78$ vs $137 / 79$, respectively; $p=$ 0.04 ), higher $\mathrm{HbA}_{1 \mathrm{c}} \%$ (8.5 vs 8.0, respectively; $p<0.001$ ), lower creatinine ( $83.3 \mathrm{vs} 87 \mu \mathrm{mol} / \mathrm{l}$, respectively; $p=0.04$ ) and lower SES $(p<0.001)$. These findings are consistent with a number of previous studies, but none of these specifically examined CVD and its risk factors according to ethnicity in a large population of individuals with type 2 diabetes [5, 16-18]. However, following adjustment for all of these factors in Cox regression, increased risk remained significant in Pakistanis. However, not all previously published studies are consistent with our findings: four reported similar or lower prevalence of CVD in Pakistanis or South Asians with 


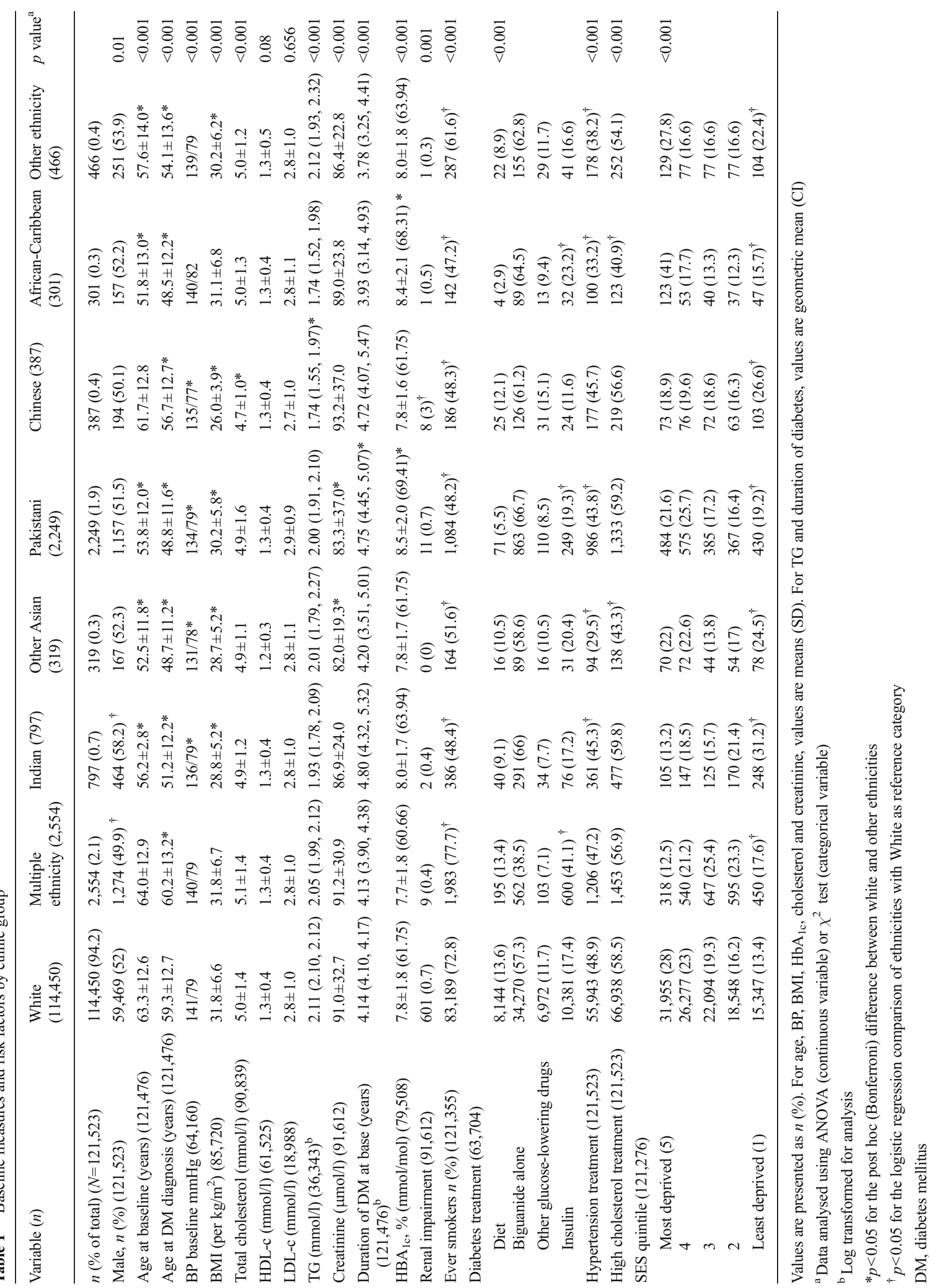




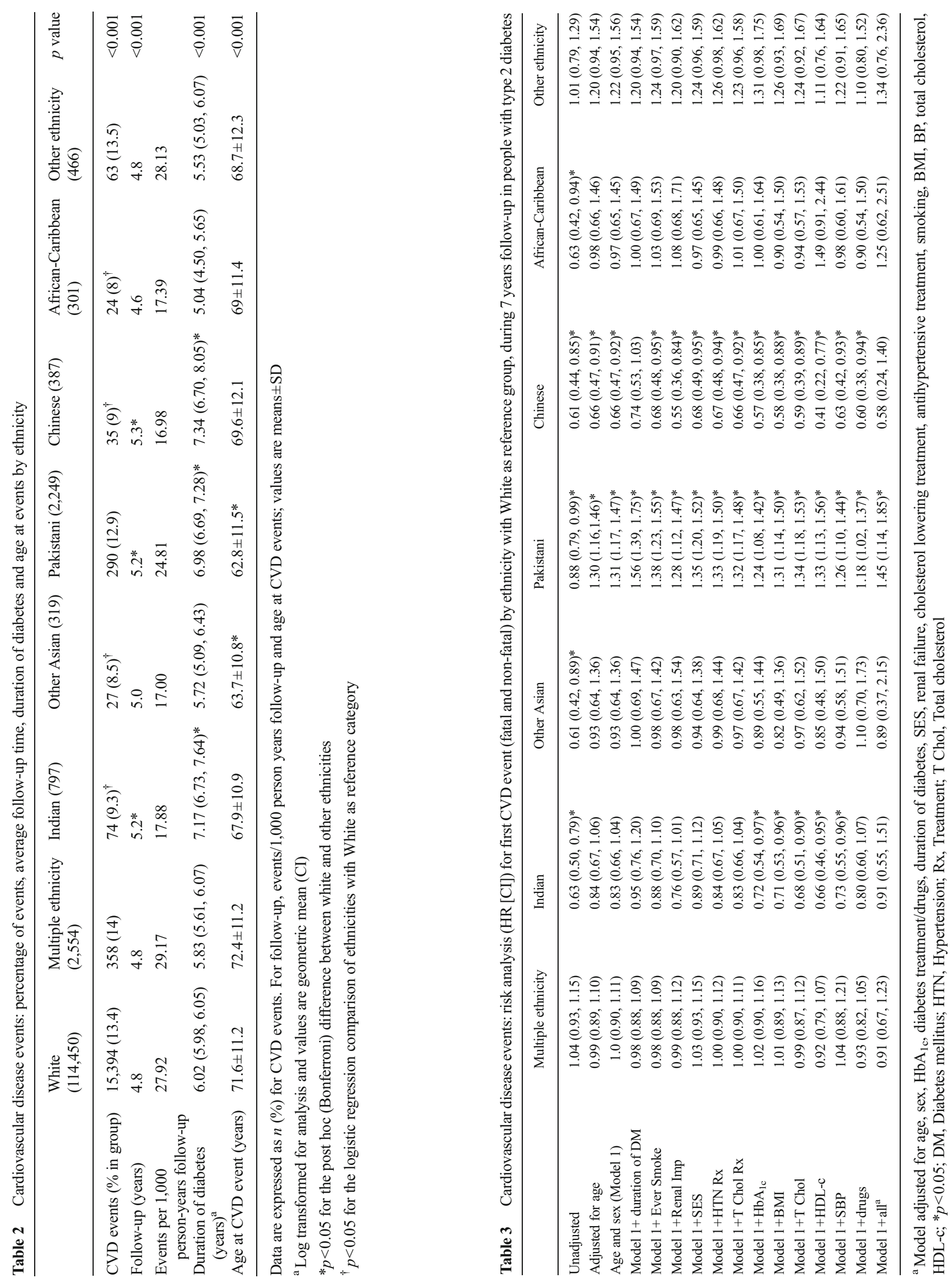



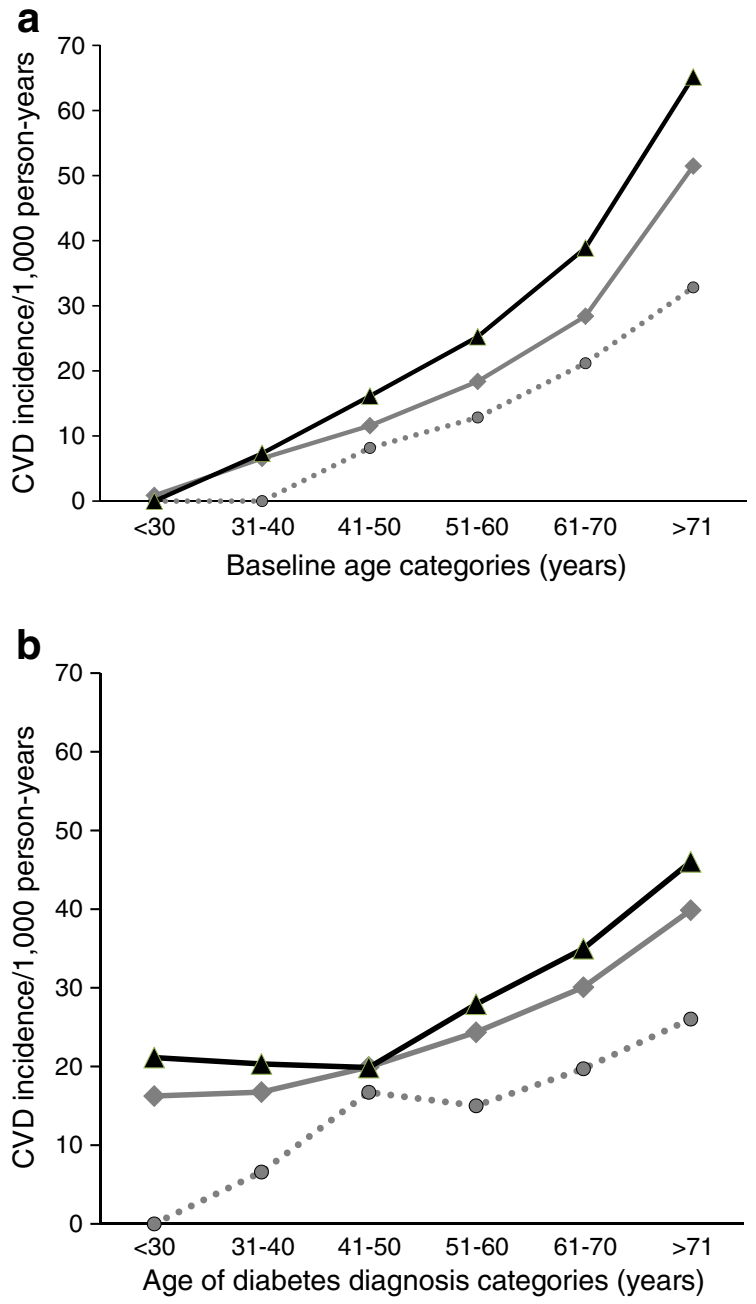

Fig. 2 Incidence of CVD per 1,000 person-years in age and age of diabetes diagnosis categories. Incidence of $\mathrm{CVD} / 1,000$ person-years in (a) baseline age categories and (b) age of diabetes diagnosis categories. Grey circles, Chinese: grey diamonds, White; black triangles, Pakistanis

diabetes as compared with whites. One of these was longitudinal but had a very small South Asian sample size of 86 patients [38]. The other three were cross sectional [16, 28, 32] and two of these reported pooled data on South Asians rather than the individual ethnicities groups [28, 32].

Pakistanis and Indians are not reported to vary largely in their genetic makeup and so the differences in cardiovascular risk reported here likely reflect other factors. It has been reported that South Asians are more likely to have cardiovascular risk factor (BMI, $\mathrm{HbA}_{1 \mathrm{c}}$, $\mathrm{BP}$, hypertension and retinopathy) evaluation and access to primary care compared with whites [14], but to date we are not aware of any such differences having been reported between Pakistanis and Indians in Scotland. Our data show that Indians are less deprived than Pakistanis but SES evaluation measures are inconsistent across different ethnic and sex groups [18] and may be a possible reason for the difference. There may be differences in access to healthcare, compliance with medication, improvement of cardiovascular risk factors over time and other health behaviours. These are interesting hypotheses that we would like to address in the future.

The population we studied included a much smaller group of individuals of Chinese origin. Our finding of a decreased risk of CVD in Chinese individuals with type 2 diabetes is also in keeping with the literature in the general population [39, 40].

Our study is one of the largest longitudinal studies of people with type 2 diabetes $(n=121,523)$ free of CVD at baseline and followed for an average of 4.8 years for the development of CVD. Other studies reporting ethnic differences were conducted in the general population and used diabetes as an adjustment factor $[8,22-24]$ rather than investigating ethnic differences among people with diabetes. Studies performed in people with type 2 diabetes were small to modest cohort studies $(n=4,974)$ [11, 12, 28, 38, 41, 42] or combined different ethnic groups (Pakistani, Indian, Bangladeshi and Sri Lankan) as one group of South Asians [8, 12, 24, 26, 28, 38, 42]. In order to avoid missing differential effects within this group, and because of the much larger size of our sample of people with type 2 diabetes, we were able to evaluate the ethnic groups Pakistani, Indian and Other Asians separately rather than merging them into one group of South Asians. This was justified by the existing literature showing ethnic differences among separate ethnic groups in the general population $[5,16$, $17,19,26]$. Most of the ethnic groups in our cohort comprised participants from one country of origin, except for Other Asians (which included Bangladeshi), and African and Caribbean blacks which were combined due to the relatively small sample size in these groups.

Strengths of this study include the population-based nature of the electronic record of diagnosed diabetes in Scotland. SCI-Diabetes has many distinguishing features such as a single shared electronic record, real time data entry (updated immediately), over $99 \%$ completeness, patient contact and care record, and anytime accessibility. SES was assessed on an area-based measure and considers several different aspects of deprivation such as employment, income, health, education, skills and training, geographic access to services, crime and housing. Individual-based measures for SES were not available but each individual area assessed using SIMD was small (median, 800 people). Patients were asked to identify their ethnic group from a standard list used in the 2001 Census in Scotland [13], which has been well used and acceptable in the general population.

One of the main limitations of our study is that 67,994 people ( $24 \%$ of total) had missing ethnicity information; and there may be a possibility that these people were less likely to have a CVD event and our results may therefore be an overestimate. Therefore, the missing ethnicity group data was 
explored and it revealed that 50,410 were free of CVD at baseline and 7,810 (15.5\%) developed CVD in the followup period giving a rate similar to that in those with known ethnicity (13.4\%). Another limitation was that we were unable to adjust our models for physical activity, diet, and family history of diabetes and CVD (which are known to vary by ethnicity) $[6,32,43]$. At the same time, the effects of many of these factors may act through BMI, BP, total cholesterol, HDL-c, hypertension and age of diabetes diagnosis, which we have adjusted for in the analysis. CVD events were coded using ICD-10 codes recorded by record linkage to SMR01. Smoking was coded as 'never smoked' and 'ever smoked' and a larger effect of smoking may have been found with a more granular measure. Another important issue is the extent to which our data are generalizable to other populations. We have stressed that considering people of South Asian origin as a single group may miss important detail. It is not possible to know whether the increased risk we have observed in those of Pakistani origin living in Scotland would be shared by people of Pakistani origin living in other countries, and therefore whether it might be explained by genetic differences, the particular characteristics of people of Pakistani origin migrating to Scotland, environmental factors or a combination of these factors.

In conclusion, Pakistani ethnicity is an independent risk factor for CVD among people with type 2 diabetes. Our finding confirms and extends existing literature demonstrating that some ethnic groups (especially South Asians) are at higher risk of CVD. This is reflected in some [44-46] but not all $[47,48]$ guidelines targeting cardiovascular risk; only one currently advises recording of ethnicity but does not recommend different specific treatment or prevention strategies [46]. The QRISK2 risk calculator (www. qrisk.org/index.php) already considers Pakistanis, Indians and other groups separately. As those of Pakistani origin had an earlier age of onset of diabetes and poorer metabolic control but apparently similar control of other risk factors such as BP, cholesterol and smoking, we suggest that programmes designed to prevent or delay onset of diabetes in this group might be of particular importance.

\footnotetext{
Acknowledgements These data are available for analysis by members of the SDRN. We thank the numerous NHS staff who enter the data and the many people and organisations (the SCI-DC Steering Group, the Scottish Diabetes Group, the Scottish Diabetes Survey Group, the managed clinical networks managers and staff in each Health Board) involved in setting up, maintaining and overseeing SCI-Diabetes. Some of the data were presented as a poster presentation at the Diabetes UK Professional Conference 2014, Liverpool [49].
}

Funding MOM was funded by Khyber Medical University, Peshawar, Pakistan. Funding for diabetes register linkage was provided by the Scottish Government. The SDRN receives financial support from NHS Research Scotland (NRS).
Duality of interest The authors declare that there is no duality of interest associated with this manuscript.

Contribution statement MOM, NS and RSL were involved in designing the study. MOM drafted the paper with contributions from RSL, JRP and NG. Analysis and interpretation was carried out by MOM with contributions from RSL, LG, HC and SW. RSL, JRP, GL, NG, LG, NS, CF, $\mathrm{HC}, \mathrm{SW}, \mathrm{RM}$ and SP were involved in data acquisition. All authors critically revised the paper and approved the final version. RSL is responsible for the integrity of the work as a whole.

\section{References}

1. The Emerging Risk Factors Collaboration, Seshasai SR, Kaptoge S et al (2011) Diabetes mellitus, fasting glucose, and risk of causespecific death. N Engl J Med 364:829-841

2. Fox CS, Coady S, Sorlie PD et al (2007) Increasing cardiovascular disease burden due to diabetes mellitus-The Framingham Heart Study. Circulation 115:1544-1550

3. Sheth T, Nair C, Nargundkar M, Anand S, Yusuf S (1999) Cardiovascular and cancer mortality among Canadians of European, south Asian and Chinese origin from 1979 to 1993: an analysis of 1.2 million deaths. Can Med Assoc J 161:132-138

4. Wild S, McKeigue P (1997) Cross sectional analysis of mortality by country of birth in England and Wales, 1970-92. Br Med J 314:705-710

5. Wild S, Fischbacher C, Brock A, Griffiths C, Bhopal R (2007) Mortality from all causes and circulatory disease by country of birth in England and Wales 2001-2003. J Public Health 29:191-198

6. Joshi P, Islam S, Pais P et al (2007) Risk factors for early myocardial infarction in South Asians compared with individuals in other countries. JAMA 297:286-294

7. Bellary S, Paul O'Hare J, Raymond NT et al (2010) Premature cardiovascular events and mortality in south Asians with type 2 diabetes in the United Kingdom Asian Diabetes Study GÇô effect of ethnicity on risk. Curr Med Res Opin 26:1873-1879

8. Forouhi NG, Sattar N, Tillin T, McKeigue PM, Chaturvedi N (2006) Do known risk factors explain the higher coronary heart disease mortality in South Asian compared with European men? Prospective follow-up of the Southall and Brent studies, UK. Diabetologia 49:2580-2588

9. Lean MEJ, Han TS, Bush H, Anderson AS, Bradby H, Williams R (2001) Ethnic differences in anthropometric and lifestyle measures related to coronary heart disease risk between South Asian, Italian and general-population British women living in the west of Scotland. Int J Obes 25:1800-1805

10. Danaei G, Finucane MM, Lu Y et al (2011) National, regional, and global trends in fasting plasma glucose and diabetes prevalence since 1980: systematic analysis of health examination surveys and epidemiological studies with 370 country-years and 2.7 million participants. Lancet 378:31-40

11. Mukhopadhyay B, Forouhi NG, Fisher BM, Kesson CM, Sattar N (2006) A comparison of glycaemic and metabolic control over time among South Asian and European patients with type 2 diabetes: results from follow-up in a routine diabetes clinic. Diabet Med 23:94-98

12. UK Prospective Diabetes Study Group (1998) Ethnicity and cardiovascular disease - the incidence of myocardial infarction in white, South Asian, and Afro-Caribbean patients with type 2 diabetes (UK Prospective Diabetes Study 32). Diabetes Care 21:1271-1277

13. Negandhi PH, Ghouri N, Colhoun HM et al (2013) Ethnic differences in glycaemic control in people with type 2 diabetes mellitus living in Scotland. PLoS One 8:e83292 
14. Fischbacher CM, Bhopal R, Steiner M, Morris AD, Chalmers J (2009) Is there equity of service delivery and intermediate outcomes in South Asians with type 2 diabetes? Analysis of DARTS database and summary of UK publications. J Public Health 31:239-249

15. McKeigue PM, Shah B, Marmot MG (1991) Relation of central obesity and insulin resistance with high diabetes prevalence and cardiovascular risk in South Asians. Lancet 337:382-386

16. Zaninotto P, Mindell J, Hirani V (2007) Prevalence of cardiovascular risk factors among ethnic groups: results from the Health Surveys for England. Atherosclerosis 195:e48-e57

17. Brindle P, May M, Gill P et al (2006) Primary prevention of cardiovascular disease: a web-based risk score for seven British black and minority ethnic groups. Heart 92:1595-1602

18. Fischbacher CM, Cezard G, Bhopal RS, Pearce J, Bansal N (2014) Measures of socioeconomic position are not consistently associated with ethnic differences in cardiovascular disease in Scotland: methods from the Scottish Health and Ethnicity Linkage Study (SHELS). Int J Epidemiol 43:129-139

19. Bhopal RS, Unwin N, White M et al (1999) Heterogeneity of coronary heart disease risk factors in Indian, Pakistani, Bangladeshi, and European origin populations: cross sectional study. BMJ 319:215-220

20. Information Service division (ISD) Scotland. Assessment of SMR01 Data 2010-2011, Scotland Report May 2012. Information Service Division (ISD) Scotland. 2014. 18-11-2014. Available from: http:// www.isdscotland.org/Health-Topics/Hospital-Care/Publications/ 2012-05-08/Assessment-of-SMR01Data-2010-2011ScotlandReport.pdf. Accessed 18 Nov 2014

21. Govan L, Maietti E, Torsney B et al (2012) The effect of deprivation and $\mathrm{HbA}_{1 \mathrm{c}}$ on admission to hospital for diabetic ketoacidosis in type 1 diabetes. Diabetologia 55:2356-2360

22. Hempler NF, Larsen FB, Nielsen SS, Diderichsen F, Andreasen AH, Jorgensen T (2011) A registry-based follow-up study, comparing the incidence of cardiovascular disease in native Danes and immigrants born in Turkey, Pakistan and the former Yugoslavia: do social inequalities play a role? BMC Public Health 11:662

23. Hippisley J, Coupland C, Robson J, Brindle P (2010) Derivation, validation, and evaluation of a new QRISK model to estimate lifetime risk of cardiovascular disease: cohort study using QResearch database. BMJ 341:1-10

24. Munter JSD, Agyemang C, Stronks K, Valkengoed IGV (2013) Association of physical activity, smoking, and alcohol intake with CVD-related hospital discharge in people of European, South Asian, or African descent. Eur J Prev Cardiol 20:80-88

25. Bradshaw P, Bromley C, Corbett J et al (2012) Cardiovascular disease, diabetes and hypertension. In: Lisa R, Clare S, Catherine B (eds) The Scottish Health Survey 2011 (Volume 1: Adults). 2012 edn. The Scottish Government, Edinburgh, pp 223-241

26. Bhopal R, Fischbacher C, Vartiainen E, Unwin N, White M, Alberti G (2005) Predicted and observed cardiovascular disease in South Asians: application of FINRISK, Framingham and SCORE models to Newcastle Heart Project data. J Public Health 27:93-100

27. Zahid N, Shi Z, Claussen B, Hussain A (2009) Prevalence and risk factors for diabetes, comparison of rural populations in Bangladesh, China and Pakistan. Diabetes Metab Syndr Clin Res Rev 3:109-112

28. Mehta RL, Davies MJ, Ali S et al (2011) Association of cardiac and non-cardiac chronic disease comorbidity on glycaemic control in a multi-ethnic population with type 1 and type 2 diabetes. Postgrad Med J 87:763-768

29. Wolffenbuttel BH, Herman WH, Gross JL, Dharmalingam M, Jiang HH, Hardin DS (2013) Ethnic differences in glycemic markers in patients with type 2 diabetes. Diabetes Care 36:2931-2936

30. Bhatnagar D, Anand IS, Durrington PN et al (1995) Coronary riskfactors in people from the Indian subcontinent living in west London and their siblings in India. Lancet 345:405-409
31. Tran AT, Straand J, Diep LM, Meyer HE, Birkeland KI, Jenum AK (2011) Cardiovascular disease by diabetes status in five ethnic minority groups compared to ethnic Norwegians. BMC Public Health 11:554

32. Mohanty SA, Woolhandler S, Himmelstein DU, Bor DH (2005) Diabetes and cardiovascular disease among Asian Indians in the United States. J Gen Intern Med 20:474-478

33. Fischbacher CM, Hunt S, Alexander L (2004) How physically active are South Asians in the United Kingdom? A literature review. J Public Health 26:250-258

34. Darr A, Astin F, Atkin K (2008) Causal attributions, lifestyle change, and coronary heart disease: illness beliefs of patients of South Asian and European origin living in the United Kingdom. Heart Lung J Acute Crit Care 37:91-104

35. Jaffiol C, Thomas F, Bean K, Jego B, Danchin N (2013) Impact of socioeconomic status on diabetes and cardiovascular risk factors: results of a large French survey. Diabetes Metab 39:56-62

36. Robbins JM, Vaccarino V, Zhang H, Kasl SV (2005) Socioeconomic status and diagnosed diabetes incidence. Diabetes Res Clin Pract 68:230-236

37. Jackson CA, Jones NRV, Walker JJ et al (2012) Area-based socioeconomic status, type 2 diabetes and cardiovascular mortality in Scotland. Diabetologia 55:2938-2945

38. Anavekar NS, Gans DJ, Berl T et al (2004) Predictors of cardiovascular events in patients with type 2 diabetic nephropathy and hypertension: a case for albuminuria. Kidney Int 66:S50-S55

39. Bansal N, Fischbacher CM, Bhopal RS et al (2013) Myocardial infarction incidence and survival by ethnic group: Scottish Health and Ethnicity Linkage retrospective cohort study. BMJ Open 3:e003415

40. Harland JO, Unwin N, Bhopal RS et al (1997) Low levels of cardiovascular risk factors and coronary heart disease in a UK Chinese population. J Epidemiol Community Health 51:636-642

41. Khuwaja AK, Lalani S, Azam IS, Ali BS, Jabbar A, Dhanani R (2011) Cardiovascular disease-related lifestyle factors among people with type 2 diabetes in Pakistan: a multicentre study for the prevalence, clustering, and associated sociodemographic determinants. Cardiol Res Pract 2011:656835

42. Mather HM, Chaturvedi N, Fuller JH (1998) Mortality and morbidity from diabetes in South Asians and Europeans: 11-year follow-up of the Southall Diabetes Survey, London, UK. Diabet Med 15:53-59

43. Holmboe-Ottesen G, Wandel M (2012) Changes in dietary habits after migration and consequences for health: a focus on South Asians in Europe. Food Nutr Res 56. doi:10.3402/fnr.v56i0.18891

44. Cooper A, O Flynn N (2008) Risk assessment and lipid modification for primary and secondary prevention of cardiovascular disease: summary of NICE guidance. BMJ 336:1246-1248

45. Greenland P, Alpert JS, Beller GA et al (2010) 2010 ACCF/AHA guideline for assessment of cardiovascular risk in asymptomatic adults: a report of the American College of Cardiology Foundation/ American Heart Association task force on practice guidelines developed in collaboration with the American Society of Echocardiography, American Society of Nuclear Cardiology, Society of Atherosclerosis Imaging and Prevention, Society for Cardiovascular Angiography and Interventions, Society of Cardiovascular Computed Tomography, and Society for Cardiovascular Magnetic Resonance. J Am Coll Cardiol 56:e50-e103

46. Scottish Intercollegiate Guidelines Network. Risk estimation and the prevention of cardiovascular disease. Guideline No 97. SIGN. 3-62014. 21-8-2014. Available from: http://www.sign.ac.uk/guidelines/ fulltext/97/. Accessed 3 June 2014

47. Woodward M, Brindle P, Tunstall-Pedoe H (2007) Adding social deprivation and family history to cardiovascular risk assessment: the ASSIGN score from the Scottish Heart Health Extended Cohort (SHHEC). Heart 93:172-176 
48. Task FM, Perk J, de Backer G et al (2012) European guidelines on cardiovascular disease prevention in clinical practice (version 2012): the fifth joint task force of the European Society of Cardiology and other societies on cardiovascular disease prevention in clinical practice (constituted by representatives of nine societies and by invited experts) developed with the special contribution of the European Association for Cardiovascular Prevention \& Rehabilitation (EACPR). Eur Heart J 33:16351701

49. Malik MO (2014) Ethnicity and risk of cardiovascular disease (CVD): 4.8 year follow-up of patients with type 2 diabetes in Scotland. Diabet Med 31(Suppl S1):76-77 